\title{
Percepções e Práticas dos Profissionais da Atenção Primária à Saúde na Abordagem sobre Drogas
}

\author{
Tamires Jordão Laport ${ }^{1}$ \\ Pedro Henrique Antunes da Costa \\ Daniela Cristina Belchior Mota \\ Telmo Mota Ronzani \\ Universidade Federal de Juiz de Fora
}

\begin{abstract}
RESUMO - Este estudo teve como objetivo analisar as percepções dos profissionais da Atenção Primária à Saúde (APS) em relação às práticas de prevenção e à abordagem ao usuário de álcool e outras drogas, levantando desafios e possibilidades da APS. Através de grupos focais, participaram 18 profissionais da APS de um município de pequeno/médio porte do estado de Minas Gerais, Brasil. Os resultados sugerem que há um discurso a favor das práticas preventivas, mas enfoque no curativismo; limitada participação dos usuários nas atividades preventivas; dificuldade na abordagem aos usuários de drogas; sobrecarga de trabalho; ausência de engajamento dos médicos; cobertura assistencial insuficiente; falta de suporte da gestão, entre outros fatores que obstaculizam o desenvolvimento das ações preventivas e interferem na abordagem aos usuários de drogas.
\end{abstract}

Palavras-chaves: droga (uso), prevenção, prevenção do abuso de drogas, atenção primária à saúde, saúde pública

\section{Perceptions and Practices of Professionals in Primary Health Care in the Approach of Drug Use}

\begin{abstract}
This study aimed to analyze the perceptions of the professionals of Primary Health Care (PHC) in relation to prevention practices and the approach to users of alcohol and other drugs, raising challenges and possibilities of PHC. Through focus groups, 18 PHC practitioners of a city of small / medium-sized state of Minas Gerais, Brazil, participated. The results suggest that there is a general opinion in favor of preventive practices, but the focus is on curative practices; limited user participation in preventive activities; difficulties in approaching drug users; work overload, lack of engagement of doctors; insufficient health care coverage; lack of support management, among other factors that hinder the development of preventive actions and the approach of drug users.
\end{abstract}

Keywords: drug usage, prevention, drug abuse prevention, primary health care, public health.

As noções de prevenção e promoção à saúde articulam-se ao moderno discurso de saúde pública e de redirecionamento das práticas em saúde, a partir das décadas de 70-80 (Czeresnia, 2003). Partindo de uma concepção ampla do processo saúde-doença e de seus determinantes, sustentam-se na constatação de que a saúde é produto de um espectro de fatores que se relacionam com a qualidade e as condições de vida (Buss, 2000, 2002).

Enquanto na 'antiga' saúde pública o foco era nas causas individuais das doenças, o novo movimento enfatiza as influências sociais e ambientais nos padrões de saúde (Oliveira, 2005) e volta-se para ações que envolvam coletividades. Importantes conferências realizadas pela Organização Mundial da Saúde (OMS) fundamentaram a base ideológica da promoção da saúde. Em 1978, na Conferência em Alma-Ata, com enfoques primordiais na promoção à saúde e na prevenção, foi divulgada a proposta da Atenção Primária à Saúde (APS) (Buss, 2000, 2002; Oliveira, 2005). E, no ano de 1986, na Conferência em Ottawa, foi formulado o marco na definição do conceito moderno de promoção da saúde, a Carta de Ottawa (Buss, 2000, 2002).

1 Endereço para correspondência: Universidade Federal de Juiz de Fora, Instituto de Ciências Humanas, Departamento de Psicologia, Rua José Lourenço Kelmer, s/n, Campus universitário, São Pedro, Juiz de Fora, MG, Brasil. CEP: 36036-900. E-mail: tamilaport@hotmail.com
Nesse contexto, as estratégias de organização dos sistemas de saúde com base na APS visam, além da racionalização de recursos, contemplar também, de maneira equitativa, as necessidades complexas que fazem parte do contexto social, político e econômico das populações. Uma das estratégias principais desse modelo caracteriza-se pelo enfoque na participação comunitária como meio de produção coletiva de saúde (Ronzani \& Stralen, 2003). Dessa forma, os serviços de APS estariam orientados para os principais problemas sanitários da comunidade, oferecendo atenção preventiva, curativa, reabilitação, e, principalmente, promovendo a saúde de acordo com as condições econômicas e valores socioculturais de cada localidade (Andrade, Barreto, \& Bezerra, 2006).

De acordo com Oliveira (2005), mudanças nos princípios da educação em saúde também foram ocasionadas. A 'nova' educação em saúde passa a ter que superar a conceituação biomédica de saúde e abranger objetivos mais amplos, uma vez que a saúde deixa de ser compreendida apenas como a ausência de doenças. Assim, na sua versão contemporânea, a educação em saúde já não se destina apenas a prevenir doenças, mas a preparar o indivíduo para uma vida mais saudável nos contextos nos quais se insere.

Nessa perspectiva, no Brasil, a Estratégia da Saúde da Família (ESF) aparece como a estratégia prioritária e o modelo substitutivo para a organização da APS. Entre seus 
princípios estão: possuir caráter substitutivo em relação à rede de atenção básica tradicional; atuação territorializada, com ações pactuadas com a comunidade dirigidas aos problemas de saúde; desenvolvimento de atividades com o foco na família e comunidade; busca pela integração com instituições e organizações sociais para o desenvolvimento de parcerias; e ser um espaço de construção de cidadania (Andrade et al., 2006; Brasil, 2006).

Dessa forma, as equipes de ESF, compostas por profissionais de diversas especialidades e saberes, apresentam uma proximidade e inserção com as famílias e comunidades, que lhes conferem uma posição ideal no foco da redução do risco de saúde e na promoção de saúde, incluindo a prevenção do abuso de álcool e outras drogas (Brasil, 2003; Ronzani, Amaral, Formigoni, \& Babor, 2008). Como bem elucidam Giovanella, Escorel e Mendonça (2003), “pretende-se instituir a equipe de saúde da família como a porta de entrada do sistema de atenção, devendo este primeiro nível estar integrado a uma rede de serviços" (p. 280).

Entende-se que os problemas relacionados à questão do uso de drogas, com suas origens multifatoriais, requerem a mobilização dessa rede de serviços e desses movimentos capazes de potencializar formas mais amplas de intervir em saúde. Assim, tratar da questão do uso abusivo e da dependência de substâncias psicoativas implica discutir não só as questões orgânicas e psicológicas, mas também os aspectos sociais, culturais e econômicos que perpassam esse fenômeno (Büchele, Coelho, \& Lindner, 2009; Pratta \& Santos, 2009).

A compreensão desses aspectos tem feito emergir, mesmo que lenta e gradualmente, uma mudança no tratamento aos usuários de drogas e revelado a importância de ações preventivas no dia-a-dia do atendimento aos usuários dos serviços de saúde. Entre outros motivos, isso se deve ao fato de que as práticas de prevenção podem ser praticadas para o alcance da promoção da saúde de uma grande parcela da população (Ronzani, Amaral, Formigoni, \& Babor, 2008).

A prevenção na área de drogas tem como objetivo evitar que os indivíduos abusem de drogas e, consequentemente, causem danos pessoais e sociais relacionados a esse abuso. A prevenção primária/universal dirige-se à população geral, a prevenção secundária/seletiva dirige-se a subgrupos específicos, especialmente populações de risco em relação ao uso de drogas e a prevenção terciária/indicada dirigese a pessoas que já apresentam sinais de abuso de drogas (Cartana, Santos, Fenili, \& Spricigo, 2004). Todos estes tipos de prevenção devem, no entanto, dar reconhecimento à dimensão ética e não moralista do consumo (Bucher, 2007).

Contudo, é sabido que, ainda assim, existe estigmatização por parte dos profissionais em relação aos usuários de drogas, havendo também uma falta de preparo e dificuldade para se lidar com o tema (Oliveira \& Ronzani, 2012; Ronzani, Higgins-Bidd, \& Furtado, 2009). Essa insuficiência na formação, voltada para a clínica e o modelo biomédico, aliada à escassez de programas para a atenção aos usuários de drogas nas equipes de ESF dificultam a realização de práticas preventivas e promocionais à saúde (Gonçalves \& Tavares, 2007; Ronzani \& Silva, 2008).

A partir disso, torna-se relevante avaliar as percepções dos profissionais que compõem a rede assistencial local e que vivenciam essa realidade em suas práticas cotidianas. No intuito de melhor compreender esse panorama, o presente estudo objetivou analisar as percepções dos profissionais da APS em relação às práticas de prevenção e à abordagem ao usuário de álcool e outras drogas, levantando desafios e possibilidades.

\section{Método}

O presente estudo caracteriza-se por ser um estudo de caso, de natureza qualitativa. Segundo Yin (2009), o estudo de caso (case study) é um método de investigação das ciências sociais utilizado quando os objetos do estudo referem-se ao 'como' e ao 'porquê', estando relacionados a um fenômeno contemporâneo em seu contexto natural e com o pesquisador tendo pouco controle sobre os acontecimentos. Trata-se de um recorte de uma pesquisa/intervenção realizada em um município brasileiro de pequeno/médio porte que visou implementar práticas preventivas sobre o uso de álcool e outras drogas em serviços da APS e Assistência Social.

As estratégias de prevenção adotadas basearam-se na Triagem, Intervenção Breve e Encaminhamento para tratamento (Screening, Brief Intervention, and Referral to Treatment - SBIRT), que fornecem a identificação precoce de problemas com o uso de drogas, tão bem quanto orientam os pacientes, podendo, assim, com o decorrer do tempo, reduzir os efeitos negativos de seu uso (Babor \& Higgins-Biddle, 2001). A SBIRT é composta pela aplicação do Alcohol, Smoking and Substance Involvement Screening Test - ASSIST e pela Intervenção Breve (IB). O ASSIST é um instrumento de triagem que visa à identificação do padrão de uso e ao consequente auxílio ao profissional de saúde na definição da estratégia de intervenção mais adequada (Humeniuk \& Poznyak, 2004). A IB trata-se de uma abordagem de educação em saúde relacionada à prevenção primária ou secundária para usuários de drogas, que visa à mudança de comportamento, através de um atendimento com tempo limitado e que pode ser realizado por profissionais de diferentes formações (Babor, Kassebaum, Grimaldi, McRee, Ahmed, Bray, 2007).

\section{Local}

O município estudado está situado no estado de Minas Gerais (MG), Brasil. Sua população, de acordo com o IBGE (2010), era de 69.757 habitantes. Ao todo, o município possui 153 estabelecimentos em sua rede assistencial de saúde, sendo 96 deles de natureza privada, 51 de administração direta do setor público da saúde e outros 06 serviços divididos entre entidades sem fins lucrativos, cooperativas e fundações privadas. Na APS, são compostas 17 equipes de ESF, sendo 15 equipes na zona urbana e duas na zona rural. A cobertura da ESF é de $92,81 \%$ da população (SAGE, 2013). Referente à rede assistencial em saúde mental e sobre álcool e outras drogas, o município possui um Centro de Atenção Psicossocial (CAPS), para transtornos mentais gerais; um Centro de Atenção Psicossocial Álcool e outras Drogas (CAPSad), que se encontrava em fase de implantação 
no momento do estudo; oito grupos de ajuda mútua (sete Alcoólicos Anônimos - AA e um Narcóticos Anônimos NA); e três comunidades terapêuticas.

\section{Participantes}

Participaram, no presente estudo, 18 profissionais da APS, sendo oito enfermeiros, seis agentes comunitários de saúde (ACS) e quatro profissionais do Núcleo de Apoio à Saúde da Família (NASF). Os NASF's, constituídos por profissionais de diversas áreas do conhecimento, foram criados pelo Ministério da Saúde em 2008 com o objetivo de apoiar e atuar em conjunto com os profissionais das equipes de ESF, compartilhando e apoiando as práticas em saúde nos territórios sob responsabilidade destas (Brasil, 2009). Devido à complexidade do uso de álcool e outras drogas e seu caráter multifacetado, com várias causas e impactos em diversos âmbitos, faz-se necessário um entendimento da situação e a abordagem ao problema a partir de uma ótica interdisciplinar, o que justifica a inclusão desses profissionais na amostra deste estudo.

\section{Coleta dos Dados}

A estratégia de coleta de dados contou com a realização de dois grupos focais semiestruturados, conduzidos pelos mesmos pesquisadores. O grupo focal 1 (GF I) teve a presença de quatro enfermeiros da ESF e quatro profissionais do NASF. O grupo focal 2 (GF II) contou com dez profissionais da ESF, sendo quatro enfermeiros e seis ACS. Para a composição dos grupos focais, em busca da compreensão das singularidades das áreas cobertas pelo município, optamos por profissionais que trabalhassem em diferentes locais.

Os roteiros desses grupos focais abarcaram questões referentes às percepções dos profissionais de saúde acerca da prevenção e abordagem ao uso de álcool e outras drogas. Além disso, os profissionais foram questionados a descrever e discutir os pontos facilitadores e dificultadores da APS e da ESF e como esses influenciam a abordagem e as ações preventivas sobre o uso drogas.

Cabe acrescentar também a observação participante dos pesquisadores durante toda a implementação do projeto. Os dados observados foram registrados em diários de campo, gerando relatórios sistemáticos. A partir dessa técnica de coleta de dados, o pesquisador faz parte, sob sua observação, de uma situação social para realizar uma investigação científica (Minayo, 2011).

\section{Análise dos Dados}

Os dados provenientes dos grupos focais foram gravados em mídia eletrônica e transcritos. Para a análise, foi utilizada a técnica de análise de conteúdo do tipo estrutural e temática. Essa é uma técnica tradicionalmente utilizada em pesquisa qualitativa e caracteriza-se por ser uma forma sistematizada e com rigor metodológico de análise de textos e entrevistas (Bardin, 2009).
A análise desdobrou-se em três fases: (a) a pré-análise foi a fase de organização inicial, com o objetivo de apreender, de uma forma global, as ideias principais e os seus significados, através da realização de leituras flutuantes do material; (b) a exploração do material empírico foi a análise propriamente dita, sendo realizado o recorte das unidades de registro, isolamento das falas em recortes a nível semântico (eixos temáticos), visando à categorização; e, (c) o tratamento dos resultados, inferência e a interpretação consistiu na transformação dos resultados brutos. Após isto, foram feitas inferências e interpretações dos dados previstas no aporte teórico da pesquisa (Bardin, 2009).

As análises e categorizações dos textos foram feitas com definição independente de categorias pelos pesquisadores, assegurando, assim, maior validade para a análise. Os dados foram tratados e analisados através do software ATLAS.ti versão 7.0, considerada uma sofisticada ferramenta na análise de dados qualitativos.

\section{Aspectos Éticos}

O projeto foi aprovado pelo Comitê de Ética em Pesquisa da Universidade de Juiz de Fora, processo no 096/2011. Todos os participantes consentiram sobre a realização da pesquisa, assinando o Termo de Consentimento Livre e Esclarecido. A coleta de dados foi realizada em horário de trabalho e conforme as possibilidades dos participantes.

\section{Resultados e Discussão}

Os resultados foram agrupados em eixos temáticos, que estão representados na Tabela 1 . O eixo 1 - "Relacionamento entre usuários e equipes de ESF" - representa a perspectiva dos profissionais no que se refere ao contato diário com os usuários do sistema, incluindo os que apresentam problemas com o uso de drogas, e como a relação destes com as equipes de ESF se estabelecem. O eixo 2 - "Percepções frente ao modelo preventivo de saúde" - relaciona-se à visão dos profissionais de saúde frente ao discurso predominante de prevenção na saúde apregoado pelas políticas públicas. Os eixos 3 - "Percepções sobre a prevenção ao uso de drogas" - e 4 - "Abordagem ao usuário de drogas" - relacionam-se às questões do uso de drogas, mais especificamente sobre as noções de prevenção concebidas por esses profissionais e como as mesmas são transformadas em estratégias para se lidar com os usuários de drogas. Por fim, os eixos 5 - "Aspectos da dinâmica do trabalho" - e 6 - "Desafios no trabalho em rede" - envolvem aspectos proeminentes do trabalho na APS/ESF brasileira, que se relacionam diretamente à abordagem aos usuários, à concepção do uso de drogas e à proposta de prevenção na saúde como um todo.

\section{Relacionamento entre Usuários e Equipes de ESF}

Em relação à incorporação das ações de prevenção na APS do município, fatores como a percepção do modelo de saúde e a receptividade dos usuários demarcaram uma 
Tabela 1: Eixos Temáticos da Análise de Conteúdo

\section{Eixo Temático}

Relacionamento entre usuários e equipes de ESF

Percepções frente ao modelo preventivo de saúde

Percepções sobre a prevenção ao uso de drogas

Abordagem ao usuário de drogas

Aspectos da dinâmica do trabalho

Desafios no trabalho em rede

realidade já demonstrada na literatura (Mitre, Andrade, \& Cotta, 2012). Foram reportadas críticas aos usuários quanto à falta de receptividade aos programas de prevenção de uma forma geral, à busca apenas por medicamentos e às demandas de cunho curativo e remediativo, além de uma postura de passividade, a partir da qual a responsabilidade pelas ações em saúde recai somente sobre profissionais. "Esse é o problema, eles criam expectativa só em cima da gente, eles não têm que fazer nada, a população não tem que fazer nada" (Entrevistado GF I). "Então, assim, eles não são receptivos aos grupos, o que me atrapalha muito na prevenção" (Entrevistado GF II).

A limitada participação dos usuários nas atividades preventivas tem sido destacada também em outros estudos (Ronzani \& Silva, 2008). Entretanto, os profissionais ressaltam que essa situação pode ser revertida com a disponibilização de medicamentos. "Ai juntava gente. Vai distribuir remédio, vai distribuir remédio, se você falar assim que vai fazer uma reunião e falar 'óh, hoje vai ter distribuição de Rivotril, Omeoprazol', óh, mais vai ser uma beleza" (Entrevistado GF I).

Para os profissionais, há um não entendimento por parte dos usuários frente aos objetivos da ESF e o modelo de atenção predominante. A procura pelos postos de saúde é, em sua maioria, para reivindicar medicamentos, atendimentos médicos, vacinas, etc., com pouca participação dos usuários nas ações de prevenção e promoção de saúde, que ficam prejudicadas (Serapioni \& Silva, 2011; Shimizu, Dytz, Lima, \& Moura, 2004). Contudo, apesar dessas críticas, os profissionais acabam orientando as suas práticas através da aceitação e legitimação dessas demandas sociais (Costa, Mota, Cruvinel, Paiva, \& Ronzani, 2013; Ronzani \& Silva, 2008). Tal aspecto pode ser mais bem visualizado na seguinte fala:

Só que o difícil é a gente trabalhar a população que as consultas não serão mais do jeito que eles querem, vai ser prevenção, não vai mais ser só remédio, eles vão, é, vai ser difíicil. Mas é porque, assim, eu digo por mim, a gente vai deixando a população mandar. (Entrevistado GF I)

Os fatores apresentados acima nos fazem questionar os conceitos e as formas de organização dos sistemas empregados pela ESF, que são planejados e concretizados pelas políticas públicas de cima para baixo, tendo a população como público alvo, mas não a tomando como agente partícipe da construção de seus conceitos e suas práticas. Isso gera uma desconexão de percepções frente às ações em saúde, em que a culpa por um possível não entendimento dos objetivos das propostas recai, com certa frequência, sobre a população usuária. Ela é responsabilizada por não entender a proposta da ESF, por só querer atendimento médico e não se interessar por ações de prevenção e promoção de saúde, mesmo com o grande histórico em que a saúde pública esteve atrelada ao assistencialismo e ainda com a hegemonia do modelo biomédico, que desconsidera as singularidades dos usuários submetendo-os à autoridade dos profissionais de saúde (Mitre et al., 2012; Paiva, Costa, \& Ronzani, 2012).

\section{Percepções Frente ao Modelo Preventivo de Saúde}

Quanto à percepção do modelo preventivo de saúde pelos profissionais há um discurso a favor de práticas preventivas, compreendidas como necessárias e orientadoras de ações. Entretanto, verifica-se que as atividades desenvolvidas pelas equipes de ESF ainda se pautam no modelo clínico de atenção à saúde, com grande parte do tempo realizando atendimento individual de demanda espontânea (Ronzani \& Silva, 2008; Shimizu et al., 2004).

No caso do ASSIST, é baseado na prevenção e a gente tem esbarrado muito na questão da parte curativa, né? É a mesma coisa que acontece com a gente no PSF no dia-a-dia, PSF a inserção dele foi de modelo preventivo e a gente atende, faz muita assistência, né? Modelo curativo. Então o ASSIST, a gente esbarrou um pouquinho nessa tecla. (Entrevistado GF I)

Alguns fatores contribuem para a manutenção desse modelo de atendimento pelas equipes da ESF, como a pressão feita pela população e o perfil atual dos profissionais, cuja maioria tem uma formação centrada no modelo clínico (Shimizu et al., 2004). Por isso, resta-nos saber até que ponto a população é não entendedora das ações propostas pelo modelo da ESF, ou se a própria cultura organizacional desses locais está direcionada pela lógica biomédica mecanicista, ainda que não seja sua proposta.

Cabe ressaltar que, com relação à APS e à ESF no Brasil, a literatura demonstra que, apesar de profissionais atribuírem grande importância às ações de prevenção e promoção de saúde, estas ainda ocorrem de forma paralela, muitas vezes através de esforços individuais (Costa et al., 2013; Ronzani \& Silva, 2008). O discurso preventivo, embora amplamente discutido na teoria e nas políticas, de uma forma geral, é colocado em prática nos serviços de saúde de forma insuficiente (Moretti-Pires, 2009). Tais fatores refletem a falta de clareza e especificidade das próprias definições do que seriam prevenção e promoção de saúde, que embora estejam referidas na política do Ministério da Saúde (Brasil, 2006) não possuem uma definição conceitual clara e estratégias objetivas de ações concretas (Ronzani \& Silva, 2008).

\section{Percepções sobre a Prevenção ao Uso de Drogas}

Deve-se também analisar como essa prevenção vem sendo compreendida e realizada pelas equipes na área de drogas. Apesar da importância conferida às práticas preventivas, estas se pautam especialmente em aspectos "higienistas" e de campanha, implementadas principalmente em momentos pontuais de educação em saúde, como por meio de palestras e de materiais educativos disponibilizados por entes governamentais (Ronzani \& Silva, 2008). 
Nesse sentido, grande parte dos depoimentos associava a prevenção ao uso de drogas de forma restrita a trabalhos na escola e com crianças e jovens. "Em nível de prevenção é isso, é trabalhar mesmo com a criança, porque a criança e o adolescente, eles chegam, em casa trazendo, né, o que eles ouviram, o que eles aprenderam" (Entrevistado GF I). "É um ponto dificil de trabalhar, por isso é melhor igual ela falou pegar aqueles que não são usuários, né?! educar! Os jovens é melhor, mais fácil"' (Entrevistado GF II). Percebe-se, dessa forma, que os profissionais sentem-se mais à vontade para realizar a prevenção universal, demonstrando maior dificuldade na abordagem aos usuários de risco ou que fazem uso abusivo de drogas.

Ademais, observou-se uma grande dificuldade por parte dos profissionais de saúde em deslocarem as suas visões e práticas dos processos de tratamento (ou seja, da dependência) em direção a uma atuação que enfocasse a promoção de saúde e a prevenção. "Mas o objetivo de vocês é aqueles que querem sair dessa vida, se tratar. Não é? Ser encaminhado a um tratamento" (Entrevistado GF I). Talvez, por isso, como apontou estudo de Moraes (2008), o componente de prevenção pareceu menos desenvolvido que o componente de tratamento.

\section{Abordagem ao Usuário de Drogas}

Quanto à abordagem aos usuários de drogas, as falas, em geral, retrataram uma dificuldade dos profissionais de saúde em relação a esse aspecto, como pode ser visto a partir da citação abaixo:

Nós estivemos em umas situações bem complicadas. Olha, você chega numa casa que você só toca no assunto, eles te respondem aquelas perguntas ali, eles já acham que você está querendo saber tudo da vida deles. Já acha que tem polícia no meio, já acham que, nossa. (Entrevistado GF II)

Essa limitação, relacionada à própria temática, corrobora o estudo de Aalto, Peruki e Seppa (2002), que retrata a dificuldade na abordagem aos usuários de drogas pelos profissionais da APS. Em parte, essas dificuldades podem aumentar pela falta de deteç̧ão precoce dos problemas relacionados ao uso de drogas nesse nível de atenção à saúde (Barros \& Pillon, 2007).

Essa dificuldade foi considerada ainda maior no que se refere ao enfoque das drogas ilícitas, ocorrendo, na maioria das vezes, omissão por parte dos usuários. " $O$ álcool e $o$ tabaco eles falam porque são drogas lícitas, os outros aí eles... alguns falam, mas outros não" (Entrevistado GF I). "Por ser as ilícitas que envolvem policia, ai tem aquele medo de envolver" (Entrevistado GF II). Também foram relatados problemas na abordagem aos usuários de drogas em áreas com grande vulnerabilidade social e com maiores índices de periculosidade, devido principalmente à existência do tráfico. Os profissionais se mostraram com receio de represálias por parte de usuários e/ou traficantes.

Alguns atéfalam, mas tem área, tem região que, se falar, causa problema. Como lá na região que eu trabalho, o traficante que comanda a área até pra você chegar você tem que pedir permissão pra chegar, ai fica dificil, até as pessoas ficam meio sem jeito de falar. (Entrevistado GF I)
Os profissionais também relacionaram fatores como a motivação do usuário de drogas para o tratamento e o acompanhamento da família, que tanto podem refletir positivamente quanto negativamente na abordagem.

A dificuldade maior também é o interesse da pessoa, porque, se a pessoa não tiver o interesse de parar, não adianta a gente querer, a gente fazer o ASSIST, a gente orientar, dar, assim, um conselho, porque, se eles não quiserem, não adianta (Entrevistado GF II).

"Eu acho que tem que trabalhar a família. A família é a base de tudo. Se a pessoa não tiver o apoio da família, então eu acho que 90\% eu acho que não tem recuperação" (Entrevistado GF I). Além disso, alguns profissionais disseram que os usuários não percebem a dimensão do problema. "Eles não vê, acredito que eles não veem, problemas de drogas, álcool, cigarro pra eles é uma coisa muito corriqueira, mas estamos lidando com problemas de saúde" (Entrevistado GF II).

Justamente por isso, jaz a necessidade das práticas de prevenção e orientação/educação em saúde, além do apoio social aos usuários de drogas no contexto da APS/ ESF. De acordo com Barros e Pilon (2007), mesmo com as dificuldades que perpassam a abordagem ao usuário de drogas, os profissionais consideram muito importante a inserção da assistência a esses usuários na ESF e percebem que o abuso de drogas está presente entre os agravos à saúde, requerendo, portanto, o cuidado e um maior suporte da gestão.

\section{Aspectos da Dinâmica do Trabalho}

Em relação à dinâmica de trabalho, as principais limitações foram a baixa motivação dos profissionais, a sobrecarga de trabalho e a falta de engajamento médico. A baixa motivação dos usuários surge como uma consequência dos problemas do próprio sistema, estando explicitamente relacionada à falta de recursos/subsídios e ao suporte/ respaldo insuficiente da gestão, como demonstra a seguinte fala: "É, poucos profissionais comprometidos, a resposta do próprio sistema" (Entrevistado GF I). Em detrimento de momentos pontuais de críticas ao descompromisso de alguns profissionais, as falas indicaram um esforço da maioria das equipes em realizar o melhor trabalho possível dentro das possibilidades, considerando a importância da ESF na prevenção e educação em saúde da população: "É a gente. Os profissionais de saúde são os principais divulgadores da educação em saúde. Então, se a gente não fizer nada, ficar só esperando alguém da esfera governamental fazer alguma coisa... Não vai sair do lugar nunca" (Entrevistado GF II).

Apesar dessas limitações, os profissionais relataram uma alta satisfação em realizar as ações de prevenção sobre drogas. Como apontou estudo de Ronzani e Silva (2008), essa satisfação também aparece como resultado da valorização, por parte da população, do serviço prestado.

Não, o resto foi ótimo, umas pessoas que a gente ajudou foi maravilhoso, e eu pude ajudar algumas da minha área, mas esses senhores eu tô encantada, eu falei com ele mesmo, eu ganhei um presente esse ano, é muito bom, é maravilhoso, é tão bom ficar dentro disso. (Entrevistado GF II) 
Em contrapartida à essa satisfação, o estudo de Barros e Pilon (2007) apresentou dados segundo os quais os profissionais da ESF percebem que pouco têm ajudado aos pacientes com problemas relacionados ao abuso e dependência de drogas. Consideram, assim, que os pacientes se beneficiam pouco da assistência oferecida.

A sobrecarga de trabalho, a falta de tempo e a priorização de outras atividades também foram apontadas como fatores que limitam/prejudicam as ações de prevenção ao uso de drogas, assim como em outros estudos relacionados à APS/ ESF no Brasil (Babor et al., 2007; Ronzani \& Stralen, 2003). "E tempo também, acho que tempo também. Sem ser um dia só pra trabalhar com isso, porque chega muita coisa todo dia, muita burocracia então não tem condições, muitos dados. Fora que temos que ficar entregando receitas, papeizinhos" (Entrevistado GF II). Atrelada a esses aspectos, a demanda de trabalho foi outro ponto dificultador/limitador da atuação dos profissionais na prevenção e abordagem ao uso de drogas. "Então o quê que acontece, a realidade nossa é que os postos de saúde, os PSF, as demandas aumentaram e não conseguimos atender, a área de cobertura, a demanda é enorme, uma coisa de doido" (Entrevistado GF I).

De forma geral, o serviço na ESF aparece sendo muito protocolar e institucionalizado, limitando a possibilidade de ações e tirando a autonomia dos profissionais. A infraestrutura, na maioria das vezes, é insuficiente, faltando profissionais, equipamentos e recursos. Os profissionais, por sua vez, encontram-se sobrecarregados, principalmente os enfermeiros, tendo que se desdobrar com as funções de cuidados e a gerência das equipes/unidades, e os agentes comunitários, devido à sua maior inserção com a comunidade, sendo intensamente demandados pela população.

Foi mencionada ainda a dificuldade de envolvimento dos médicos em atividades preventivas, assim como apontado por outros estudos (Costa et al., 2013; Ronzani, Mota, \& Souza, 2009; Serapioni \& Silva, 2011). Os profissionais acreditam que os médicos possuem uma grande credibilidade perante os usuários e a comunidade e que, por isso, poderiam ajudar no processo de implementação das atividades preventivas. "Porque os usuários são apaixonados pelos médicos, né, igual tem reunião, 'ah, o médico tá ai?' 'Então, nossa, eles iam ajudar muito na prevenção em todos os contextos que a gente trabalha" (Entrevistado GF I). Dessa forma, a participação da categoria profissional médica foi problematizada a fim de um maior envolvimento/cooperação desses profissionais no contexto de trabalho integrado na ESF e, por conseguinte, na prevenção ao uso de drogas.

\section{Desafios no Trabalho em Rede}

As ações integradas e a parceria entre os setores foram apontados como fundamentais para o desenvolvimento de um trabalho efetivo na abordagem do usuário de drogas. Como tentativas de ações nesse sentido, foram apontadas parcerias com escolas, com o Centro de Referência Especializado de Assistência Social (CREAS) e com o CAPSad. "Eu, tipo assim, procurei o CAPSad, procurei e vi que lá tem apoio sim, então se as pessoas forem lá, mas eu só achei assim, podia ser mais articulado da parte deles com a gente, mas também é porque é novo aqui" (Entrevistado GF II). As escolas, como mencionado anteriormente, apareceram como um espaço privilegiado para a realização de trabalhos educativos e conscientizadores, devido também à proximidade de algumas equipes de ESF com esses dispositivos. Contudo, a infraestrutura aparece novamente como uma limitação para a realização das práticas educativas e preventivas, como demonstra a fala a seguir:

Então o que a gente procura é mostrar outra realidade pra eles, pra que eles possam ter opção, tá tendo oficina de leitura, porque as crianças vão mal na escola também, né? E tem dança, tem artes, e agora a gente tá tentando inserir outras coisas, na sexta-feira tem esportes, tem informática, e nós estamos inserindo, e vamos inserir no ano que vem outras coisas, que é aula de violão, aula de capoeira, mas nós estamos dependendo do espaço, que nós não temos. (Entrevistado GF I)

Em suma, a rede intersetorial do município se mostrou ainda em processo de construção. A cobertura assistencial é insuficiente, com dificuldades para o encaminhamento de dependentes e a realização de parcerias em projetos de prevenção aos jovens. Foi observado também no município um desconhecimento dos profissionais de saúde sobre serviços e propostas que não trabalhassem com a medicação, como Centros de Convivências, espaços de lazer e de esporte no próprio território, dados esses que se coadunam com a literatura na área (Gomes, 2006). Também não houve referência sobre a criação ou o desenvolvimento de uma rede comunitária por parte dos profissionais, corroborando os achados do estudo de Moraes (2008).

As ações voltadas para a articulação da rede são válidas e extremamente necessárias. É importante ressaltar a impossibilidade de setores isoladamente responderem aos diversos fatores relacionados à problemática das drogas. Contudo, essas ações devem ser formuladas e implementadas em conjunto, levando em consideração o quantitativo de atores que fazem parte dos serviços, da gestão, mas também das comunidades. Nesse sentido, faz-se necessária a superação de práticas fragmentadas, que reverberam na carência e até mesmo na ausência de diálogo e interação entre os diferentes atores e setores que constituem a rede assistencial sobre drogas (Paiva et al., 2012).

\section{Considerações Finais}

O presente artigo objetivou analisar as percepções de profissionais da APS de um município brasileiro de pequeno/médio porte em relação às práticas de prevenção e à abordagem ao usuário de álcool e outras drogas. Ressaltamse as limitações do estudo, referentes à própria metodologia empregada, ao não abarcar as concepções dos outros atores que compõem a APS, como os usuários dos serviços e a gestão municipal.

De forma geral, pode-se dizer que a demanda para a prevenção e abordagem aos usuários de drogas é frequente e que os profissionais se mostraram atentos para a necessidade da realização de ações que englobem a complexidade dessa temática. Entretanto, essas concepções demonstram um cenário multifacetado, sob a influência direta de diversas conjunturas e aspectos que limitam/restringem a atuação 
desses profissionais, tais como a própria magnitude/ amplitude do problema, a formação insuficiente para atender a demanda, as dificuldades na relação com os usuários, a insuficiência de recursos (como infraestrutura, subsídios e suporte da gestão), dentre outros.

Além disso, abordar a temática do álcool e de outras drogas envolve em seu cerne uma complexidade de questões que perpassam desde a prevalência de concepções moralistas e reducionistas; a multidimensionalidade do fenômeno, e que, portanto, requer ações conjuntas para seu enfrentamento; a falta de preparo na formação dos profissionais que lidam diretamente com o assunto e a falta de diálogo das políticas públicas com profissionais e a população, acarretando em desconhecimento por parte dos usuários acerca das atividades voltadas para a prevenção e promoção à saúde.

A educação em saúde e as estratégias preventivas têm o papel importante de induzir novas práticas nos serviços de saúde e de facilitar a participação de importantes atores sociais no processo de construção das ações integradas sob a perspectiva ampliada de saúde. Dessa forma, devem ir além da mera transmissão de conhecimentos técnicos, propiciando uma valorização da comunidade e do usuário, fazendo ver aos profissionais o caráter integrativo das ações de saúde (Albuquerque \& Stotz, 2004).

Contudo, muitas questões devem ser exploradas para a potencialização dos processos formativos/educativos e o fortalecimento da abordagem ao uso de drogas, como, por exemplo: (a) a necessidade de mais esforços formais de capacitação profissional através de abordagens planejadas, em associação com a realidade local e objetivando a construção de ações coletivas; (b) o envolvimento dos atores sociais na construção e formulação das ações em um processo reflexivo; (c) um maior suporte da gestão municipal; (d) a regulamentação de diretrizes políticas mais específicas e objetivas no intuito de viabilizar melhor a consolidação de projetos locais.

Para a reversão desse cenário, a análise das percepções e expectativas dos atores que compõem a APS contribui para a identificação de obstáculos que afetam o cotidiano do sistema de saúde. Além disso, contribui para a elaboração de um planejamento de ações das ESF a partir do contexto social nos quais estão inseridas, discutindo-se aspectos como o funcionamento da rede, as práticas de saúde, a participação social, dentre outros.

Considera-se que a construção das ações e políticas de saúde sobre álcool e outras drogas ocorre na prática através dos atores que a conformam, a partir de seus processos interativos. Por isso, a compreensão de como se dá essa conformação através das percepções sobre a APS, favorece o planejamento de políticas que vão ao encontro da realidade, resultando em ações e serviços mais contextualizados. Quem sabe assim essas condições impliquem um horizonte promissor na prevenção e assistência aos indivíduos com problemas relacionados ao abuso de drogas e no subsídio para implementação de políticas públicas específicas.

\section{Referências}

Aalto, M., Pekuri, P., \& Seppä, K. (2002). Primary health care professional activity in intervining in patients alcohol drinssing: A patient perspective. Drug and Alcohol Dependence, 66, 39-43.

Albuquerque, P. C., \& Stotz, E. N. (2004). A educação popular na atenção básica à saúde no município: em busca da integralidade. Interface - Comunicação, Saúde e Educação, 8(15), 259-74.

Andrade L. O. M., Barreto I. C. H. C., \& Bezerra, R. C. (2006). Atenção primária à saúde e estratégia saúde da família. In Campos G. W. S., Minayo, M. C., Akerman, M. D., Drumond, J. M., Carvalho, Y. M. (Orgs). Tratado de saúde coletiva, p. 783-836. São Paulo: Editora Hucitec/Rio de Janeiro: Editora Fiocruz.

Babor, T. F., \& Higgins-Biddle, J. (2001). Brief intervention for hazardous and harmful drinking. a manual for primary care. World Health Organization: Geneva.

Babor, T. F., Mcree, G. B., Kassebaum, M. A., Grimaldi, P. L., Ahmed, K., \& Bray, J. (2007). Screening, Brief Intervention, and Referral to Treatment (SBIRT): Toward a Public Health Approach to the Management. Substance Abuse, 28(3), 7-30.

Bardin, L. (2009). Análise de Conteúdo. Lisboa: Edições 70, LDA.

Barros, M. A., \& Pillon, S. C. (2007). Assistência aos usuários de drogas: a visão dos profissionais do programa saúde da família. Revista de Enfermagem, UERJ, 15(2): 261-266.

Brasil (2006). Política Nacional de Atenção Básica. Brasília: Ministério da Saúde.

Brasil. (2003). Saúde mental e atenção básica o vínculo e o diálogo necessários. Brasília, Ministério da Saúde.

Brasil. (2009). Diretrizes do NASF - Núcleo de Apoio a Saúde da Família. Brasília: Ministério da Saúde MS.

Büchele, F., Coelho, E. B. S., \& Lindner, S. R. (2009). A promoção da saúde enquanto estratégia de prevenção ao uso das drogas. Ciência e Saúde Coletiva, 14(1), 267-273.

Bucher, R. (2007). A ética da prevenção. Psicologia: Teoria e Pesquisa, 23, 117-123.

Buss, P. M. (2000). Promoção da saúde e qualidade de vida. Ciência e Saúde Coletiva, 5(1), 163-177.

Buss, P. M. (2002). Promoção da Saúde da Família. Revista Brasileira de Saúde da Família, 2(6), 50-63.

Cartana, M. H. F., Santos, S. M. A., Fenili, R. M., \& Spricigo, J. S. (2004). Prevenção do uso de substâncias psicoativas. Texto e Contexto Enfermagem, 13(2), 286-289.

Costa, P. H. A., Mota, D. C. B. M., Cruvinel, E., Paiva, F. S., \& Ronzani, T. M. (2013) Metodologia de implementação de práticas preventivas ao uso de drogas na atenção primária latino-americana. Revista Panamericana de Saúde Pública, 33(5), 325-331.

Czeresnia, D. (2003). O conceito de saúde e a diferença entre prevenção e promoção. In D. Czeresnia, \& C.M. Freitas. (Orgs.), Promoção da saúde: Conceitos, reflexões, tendências (pp. 39-53). Rio de Janeiro: Fiocruz.

Giovanella, L., Escorel, S., \& Mendonça M. H. M. (2003). Porta de entrada pela atenção básica? Integração do PSF à rede de serviços de saúde. Saúde Debate, 27(65), 278-289.

Gomes, V. G. (2006). Apoio Matricial: Estratégia de interlocução na rede de saúde de Campinas/SP. Trabalho de conclusão do curso de Aprimoramento em Saúde Mental da Faculdade de Ciências Médicas, Universidade Estadual de Campinas, São Paulo. 
Gonçalves, S. S. P. M., \& Tavares, C. M. M. (2007). Atuação do enfermeiro na atenção ao usuário de álcool e outras drogas nos serviços extra-hospitalares. Revista de Enfermagen, 11(4), 586-592.

Humeniuk, R., \& Poznyak, V. (2008). ASSIST. Teste de triagem para álcool, tabaco e substâncias: Guia para uso na atenção primária à saúde: Versão preliminar 1.1. São Paulo, OMS.

Instituto Brasileiro de Geografia e Estatística. (2010).Cidades@. Recuperado de: $<$ http://www.ibge.gov.br/cidadesat/topwindow. htm?1>

Minayo, M. C. S. (2011). Trabalho de campo: contexto de observação, interação e descoberta. In M. C. S. Minayo (Org), Pesquisa Social: Teoria, método e criatividade, p. 61-77. Petrópolis: Vozes, 2011.

Mitre, S. M., Andrade, E. I. G., \& Cotta, R. M. M. (2012). Avanços e desafios do acolhimento na operacionalização e qualificação do Sistema Único de Saúde na Atenção Primária: Um resgate da produção bibliográfica do Brasil. Ciência e Saúde Coletiva, 17(8), 2071-2085.

Moraes, M. (2008). O modelo de atenção integral à saúde para tratamento de problemas decorrentes do uso de álcool e outras drogas: Percepções de usuários, acompanhantes e profissionais. Ciência e Saúde Coletiva, 13(1), 121-133.

Moretti-Pires R. O. (2009). Complexidade em Saúde da Família e formação do futuro profissional de saúde. Interface Comunicação, Saúde e Educação, 13(30), 153-66.

Oliveira, D. L. (2005). A 'nova' saúde pública e a promoção da saúde via educação: Entre a tradição e a inovação. Revista Latino-Americana de Enfermagem, 13(3), 423-431.

Oliveira, M. C., \& Ronzani, T. M. (2012). Estigmatização e prática de profissionais da APS referentes ao consumo de álcool. Psicologia: Ciência e Profissão, 32(3), 648-661.

Paiva, F. S., Costa, P. H. A., \& Ronzani, T. M. (2012). Fortalecendo redes sociais: Desafios e possibilidades na prevenção ao uso de drogas na atenção primária à saúde. Aletheia, 37(1), 57-72.
Pratta, E. M. M., \& Santos, M. A. (2009). O processo saúde-doença e a dependência química: interfaces e evolução. Psicologia: Teoria e Pesquisa, 25(2), 203-211.

Ronzani, T. M., Amaral, M. B., Formigoni, M. L. O. S., \& Babor, T. F. (2008). Evaluation of a training program to implement alcohol screening, brief intervention and referral to treatment in primary health care in Minas Gerais, Brazil. Nordic Studies on Alcohol and Drugs, 42, 529-538.

Ronzani, T. M., Higgins-Biddle, J., \& Furtado, E. F. (2009). Stigmatization of alcohol and other drug users by primary care providers in Southeast Brazil. Social Science \& Medicine, 69(7), 1080-1084.

Ronzani, M. T., Mota, D. C. B., \& Souza, I. C. W. (2009). Prevenção do uso de álcool na atenção primária em municípios do estado de Minas Gerais. Revista de Saúde Pública, 43(Supl.1), 51-61.

Ronzani, T. M., \& Silva, C. M. (2008). O Programa Saúde da Família segundo profissionais de saúde, gestores e usuários. Ciência e Saúde Coletiva, 13(1), 23-34.

Ronzani, T. M., \& Stralen, C. J. V. (2003). Dificuldades de implementação do Programa de Saúde da Família como estratégia de reforma do sistema de saúde brasileiro. Revista de APS, 6(2), 99-107.

SAGE. (2013). Atenção à Saúde. Recuperado de: < http://189.28.128.178/sage/>.

Serapioni, M., \& Silva, M. G. C. (2011). Avaliação da qualidade do Programa Saúde da Família em municípios do Ceará. Uma abordagem multidimensional. Ciência e Saúde Coletiva, 16(11), 4315-4326.

Shimizu, H. E., Dytz, J. L. G., Lima, M. G., \& Moura, A. S. (2004). A prática do auxiliar de enfermagem do programa saúde da família. Revista Latino-Americana de Enfermagem, 12(5), 713-20.

Yin, R. K. (2009). Case study research: design and methods (4 ed.). SAGE Publications: Londres.

Recebido em 01.08.2013

Primeira decisão editorial em 26.02.2015

Aceito em 26.02.2015 